\title{
KULTURELLE HETEROGENITÄT ALS HERAUSFORDERUNG IN BENINISCHEN ALLGEMEINBILDENDEN SEKUNDARSCHULEN
}

\author{
Sèlognon Nonvide ${ }^{1}$, \\ Vincent Atabavikpo ${ }^{2 i}$ \\ ${ }^{1}$ Inspecteur de l'enseignement secondaire, \\ Benin \\ ${ }^{2}$ Université d'Abomey-Calavi, \\ Benin
}

\begin{abstract}
:
The present study focused on the management of cultural diversity and the development of intercultural competences in secondary education has made it possible to explore the ethnocultural realities of CEG Banikanni and Tohouè in Benin. This realities are characterized by a plurality of ethnic groups, mother tongues and religions. It has also shown that good management of school life depends on taking religious, ethnic, linguistic and gender factors into account. A. D. Frederick quotes Dillard in the following terms : "Providing an appropriate education for students from diverse ethnic and cultural backgrounds is not an easy task for the classroom teacher." However, it is clear that in Benin, the development of intercultural competence is lacking in the training of trainee teachers and school authorities.
\end{abstract}

Keywords: management, intercultural, ethnocultural, diversity, competence

\section{Resume:}

La présente étude sur la gestion de la diversité culturelle et le développement des compétences interculturelles à l'enseignement secondaire a permis d'explorer les réalités ethnoculturelles des CEG Banikanni et Tohouè. Ces réalités sont caractérisées par une pluralité d'ethnies, de langues maternelles et de religions. Elle a aussi permis de parvenir aux résultats que la bonne gestion de la vie scolaire dépend de la prise en compte des facteurs religieux, ethniques, linguistiques, et de la question genre. A. D. Frederick cite Dillard en ces termes : "Donner une éducation appropriée à des élèves d'origines ethniques et culturelles diverses n'est pas une tâche aisée pour l'enseignant en classe. » Or, force est de constater que le développement de la compétence interculturelle fait défaut dans la formation des élèves-professeurs et des responsables administratifs des établissements scolaires.

Mots-clés: management, interculturel, ethnoculturel, diversité, compétence

iCorrespondence: email episel2013@gmail.com, vincentdjim65@yahoo.fr 


\section{Resumee:}

Die vorliegende Studie zum Management kultureller Vielfalt und zur Entwicklung interkultureller Kompetenz in den Sekundarschulen hat es ermöglicht, die ethnokulturellen Realitäten der CEG Banikanni und Tohouè zu erforschen. Diese Realitäten zeichnen sich durch eine Vielzahl von ethnischen Gruppen, Muttersprachen und Religionen aus. Die Studie hat auch gezeigt, dass das gute Management des Schullebens davon abhängt, religiöse, ethnische, sprachliche und geschlechtsspezifische Faktoren zu berücksichtigen. A. D. Frederick zitiert Dillard, der betont, dass es für die Lehrperson keine leichte Aufgabe sei, Lernenden aus verschiedenen ethnischen und kulturellen Hintergründen eine angemessene Ausbildung zu geben. Es ist jedoch festzustellen, dass in Benin die Entwicklung interkultureller Kompetenz bei der Ausbildung von Lehramtsstudenten und Verantwortlichen der Schulverwaltung fehlt.

Schlüsselwörter: Management, interkulturell, ethnokulturell, Vielfalt, Kompetenz

\section{Einführung}

Das beninische Schulsystem ist sich dessen bewusst, dass in der Schule "Vielfalt statt Einfalt" (Ingrid Ahlring 2002: 8) herrscht. Das bedeutet, dass in einer Schule eine Vielfalt von Persönlichkeiten, Ethnien, religiösen Gruppen, Leistungspotenzialen etc. existiert. Unabhängig von ihren religiösen Vorstellungen, Ethnie, Kultur oder Sprachzugehörigkeit unterliegen die Lernenden derselben Schulgesetzgebung und derselben Schulordnung, auch wenn ihre soziokulturelle Herkunft und ihre religiösen Praktiken unterschiedlich sein mögen.

In einem solchen Umfeld werden die Lehrenden und die Schulverwaltung beim Umgang mit der kulturellen Vielfalt mit Problemen konfrontiert: Es wird schwierig, Schulstandards anzuwenden. Was als ein Fehler angesehen wird, könnte für einen Lernenden gemäß seinen Bräuchen und sozialen Praktiken als normal angesehen werden. Darüber hinaus haben die Lehrkräfte oft kein interkulturelles Training erhalten, um die kulturelle Vielfalt, die die städtischen und ländlichen Gebiete im Allgemeinen und das Schulumfeld ins Besondere ausmacht, am besten zu bewältigen. Die kulturelle Mischung und die kulturelle Vielfalt, die die drei größten Städte von Benin prägen, wirken sich zwangsläufig auf das Schulleben der Mittelund Oberschulen dieser Städte aus. Aus diesen Gründen haben wir uns dafür entschieden, diese Auswirkungen in CEG Banikanni in Parakou und in CEG Tohouè, eine Grenzortschaft zu Nigeria, zu untersuchen. Eine Feldforschung an den oben erwähnten Orten, hat es uns ermöglicht, Daten mit Fragebogen zusammenzustellen, die einer genaueren Analyse unterzogen werden. So wird in dem ersten Teil der Untersuchung auf die Frage der Identität der Schüler und dann der Lehrer eingegangen, ehe die Problematik der guten Integration der Vielfalt in Bezug auf Religionen, Ethnien und Gender gründlich angesprochen wird. 


\section{Zur Identitätsfrage der Befragten}

\subsection{Zur Identität der Schülerschaft}

Die befragten Lernenden haben ein Durchschnittsalter von 21 Jahren, der älteste Lernende ist 26 Jahre alt und der jüngste 16. Wie die folgenden Statistiken zeigen, sind die Jungen in der Mehrheit: Von insgesamt 100 befragten Lernenden gibt es 64 Jungen und 36 Mädchen, was jeweils $64 \%$ und $36 \%$ der befragten Lernenden entspricht. Die Mehrheitsethnien sind die Fon (23,5\%), die Nago (10,2\%), die Baatsnum (8,2\%), die Defi $(8,2 \%)$, die Yoruba $(7,1 \%)$, die Maxi $(7,1 \%)$, die Gùn $(6,1 \%)$, die Lokpa $(5,1 \%)$ und die Otamari $(5,1 \%)$. Folgende Ethnien sind in der Minderheit : die Ife (1\%), die Dendi (1\%), die Nateni (1\%), die Saxwue (1\%), die Aja (1\%), die Mina (1\%), die Setっ (1\%), die Kotafon (1\%), die Tofin (1\%), die xawsa (2\%), die Pullo: $(2 \%)$, die Idaaca (3\%) und die Xwla (3\%). Die Muttersprachen der befragten Lernenden sind : Fon (27,6\%), Gùn (13,3\%), Baatsnum (8,2\%), Lokpa (6,1\%), Yoruba (6,1\%), Dendi (5,1\%), Nago (5,1\%), Defi (5,1\%), Ditamari $(4,1 \%)$, Idaaca (3,1\%), Cabe (3,1\%), Maxi (3,1\%), Xwla (2\%), Pullo (2\%), Nateni (1\%), Saxwue $(1 \%)$, Diula (1\%), Yende (1\%), Ewe (1\%) und Tofin (1\%). Die Lernenden praktizieren entweder das Christentum (78,8\%) oder den Islam (21,2\%).

Diese Statistiken bestätigen die ethnische bzw. kulturelle Heterogenität der Klassen in Benin: Für die fünf untersuchten Klassen können 22 ethnische Gruppen, 20 Muttersprachen und mindestens sieben Religionen (die muslimische, katholische, protestantische, "Chritianisme céleste-", evangelikale Religion und andere weitere christliche Religionen) identifiziert werden.

\subsection{Zur Identität der Lehrkräfte}

Von den 30 befragten Lehrenden werden 26 Männer und 4 Frauen gezählt. Die Lehrenden sind wie die Lernenden unterschiedlicher ethnischer Herkunft. 13 Ethnien sind identifiziert worden: die Gùn (23,3\%), die Fon (16,\%), die Baatınum (13,3\%), die Nago (10\%), die Xwla (10\%), die Boo $(3,3 \%)$, die Waci (3,3\%), die Waama (3,3\%), die Idaaca (3,3\%), die Mina (3,3\%), die Defi $(3,3 \%)$, die Tori (3,3\%) und die $A j a(3,3 \%)$. Es gibt 17 Muttersprachen: Acht Lehrende sprechen Gùn (26,7\%), drei Fon (10\%), zwei Maxi (6, 7\%), zwei Baatonum (6,7\%), zwei Dendi (6,7\%), ein Waci (3,3\%), ein Boo (3,3\%), ein Waama (3,3\%), ein Idaaca (3,3\%), ein Nago (3,3\%), ein Cabe (3,3\%), ein Xwla (3,3\%), ein $A j a(3,3 \%)$, ein Mina (3,3\%), ein If $\varepsilon$ (3,3\%), ein Defi (3,3\%) und ein Ayizs (3,3\%). Die befragten Lehrenden praktizieren entweder das Christentum (80\%) oder den Islam (16,7\%). zehn Lehrende behaupten, Katholiken zu sein, sechs Christen, fünf Muslime, fünf Protestanten, zwei Evangelikale, ein „Céleste“ und ein Methodist.

Bei Lernenden als auch bei Lehrenden gilt der Animismus als heikles Thema. Diskriminierende Handlungen in den Schulen sind auf die Zugehörigkeit zum Animismus zurückzuführen, wie ein befragter Lernender feststellte. Wer sich als Anhänger des Animismus in der Schule zeigt, ist sofort Opfer des Spotts. Außerdem haben die Schulbehörden von CEG Tohoué in dem im Anhang stehenden Interview (S. xix) gesagt, sie seien gelegentlich mit dem Problem der Lernenden konfrontiert, die Anhänger der Wassergöttin fallen manchmal in Trance. Diese erwähnten Fakten zeigen, dass manche Schüler tatsächlich den Animismus praktizieren. Aber oft verzichten einige Lernende und Lehrende darauf, ihre religiöse Identität anzugeben und ziehen sich lieber in die Religionen, Sprachen und sogar Ethnien zurück, mit denen sie es 
vermeiden, dem Hohn ihrer Kameraden ausgesetzt zu werden. A. Maalouf (1998:34) erklärt die Gründe für diese Einstellung zur Identität wie folgt:

"On a souvent tendance à se reconnaître dans son appartenance la plus attaquée; parfois, quand on ne se sent pas la force de la défendre, on la dissimule, alors elle reste au fond de soi-même, tapie dans l'ombre, attendant sa revanche; mais qu'on l'assume ou qu'on la cache, qu'on la proclame discrètement ou bien avec fracas, c'est à elle qu'on s'identifie. L'appartenance qui est en cause - la couleur, la religion, la langue, la classe... - envahit alors l'identité tout entière. »

Diese Anführungen deuten darauf hin, dass die kulturelle Vielfalt der Schülerschaft zu gewaltigen Schwierigkeiten führt.

\section{Die Berücksichtigung der Religions-, Ethnien- und Gendervielfalt als effiziente interkultureller Einsatz}

\subsection{Die Berücksichtigung der religiösen Vielfalt als Kriterium eines guten Managements des Schullebens}

Die meisten der befragten Lernenden (63\%) denken, dass die Schulordnung nicht im Einklang mit ihren jeweiligen kulturellen Werten und Praktiken stehen. Auf die gleiche Weise glauben sie, dass die Lehrenden und die Aufsichtsbehörden sie für Taten bestrafen, die ihrer Meinung nach mit ihren religiösen Vorstellungen und Praktiken übereinstimmen. 48\% der befragten Schüler bestätigen dies ganz deutlich und 13\% weniger kategorisch. Die genannten Gründe für die Bestrafungen sind die folgenden: das Tragen von extravaganten Frisuren und Zöpfen, das Tragen vom Bart, vom Schleier, vom Hut, vom Rosenkranz, vom Kopftuch und vom Kruzifix. Die Eltern (5\%) greifen aber selten ein, um die kulturellen und religiösen Praktiken ihrer Kinder in der Schule zu rechtfertigen. Obwohl die Lernenden gezwungen sind, sich an die Schulordnung zu halten, sagen viele Lernende $(82 \%)$, sie würden sich in der Schule nicht fremd fühlen, sie seien vielmehr mit Schulkameraden verschiedener Glaubensrichtungen befreundet und würden sich von Lehrenden oder Mitschülern wegen ihrer Religionszugehörigkeit nicht ausgeschlossen fühlen. Die Mehrheit der befragten Lernenden, unabhängig von ihrer Religionszugehörigkeit, ist der Ansicht, dass die Einschulung von Mädchen von entscheidender Bedeutung ist. Darüber hinaus fühlen sich die meisten Lernenden (72\%) nicht beleidigt durch Spitznamen im Zusammenhang mit ihrer Religion und $54 \%$ denken, von ihren Lehrenden fair behandelt zu werden. Hingegen sagen nur $40 \%$ der Lernenden sehr deutlich, dass die Aufsichtsbehörden sie genauso behandeln wie andere Lernende. 


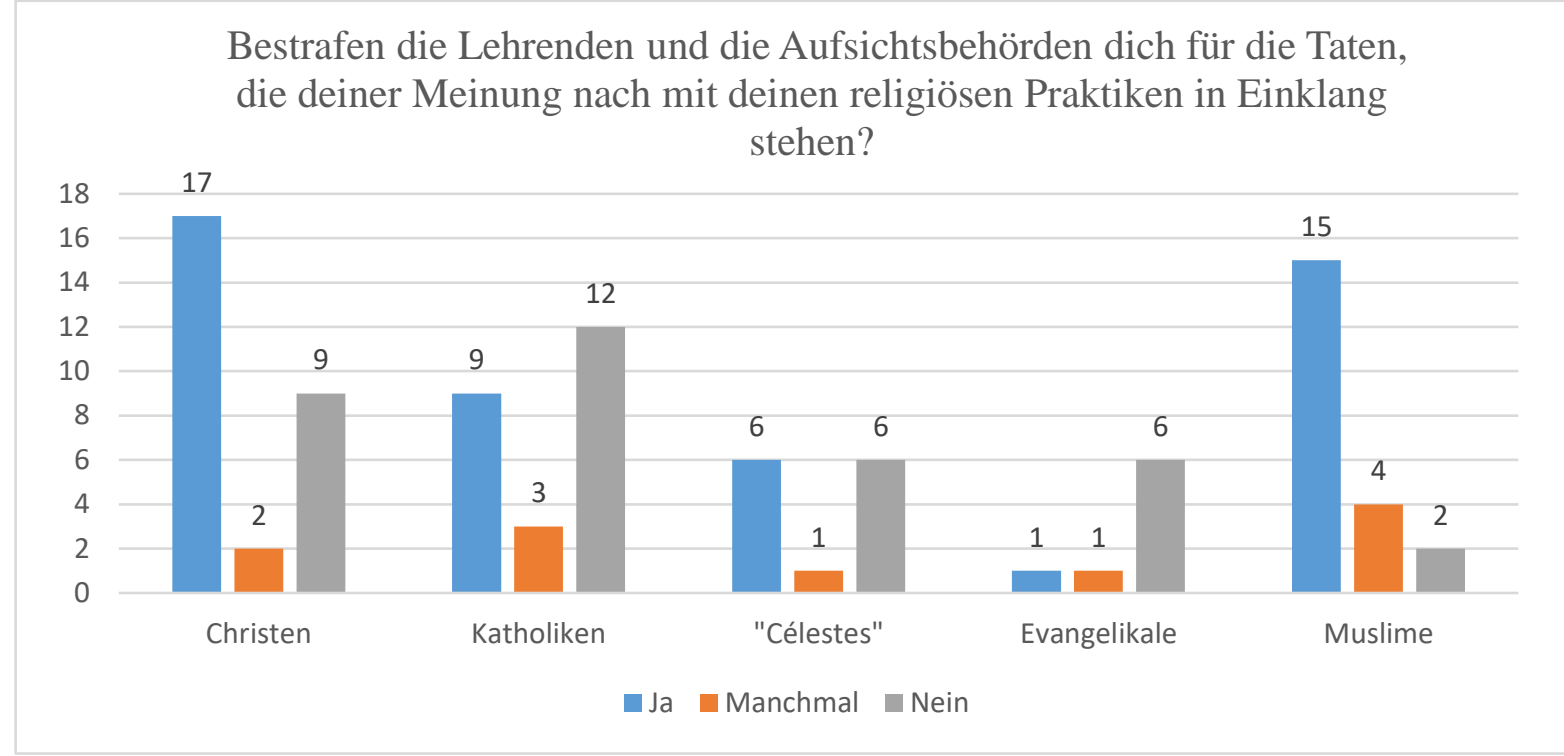

Abbildung 1: Ergebnis der Frage 6 an die Lernenden

Mit den angegebenen Gesamtstatistiken sehen wir, dass die beiden Zielschulen der vorliegenden Studie ein günstiges Umfeld für religiöse Vielfalt sind. Wir haben aber, wie schon erwähnt, einige Frustrationsstimmen seitens der Lernenden registriert, insbesondere bezüglich der Art und Weise, wie die Aufsichtsbehörden sie in Bezug auf ihre kulturellen Praktiken behandeln. Ebenfalls bestreiten die meisten Lernenden die Angemessenheit der Schulordnung mit ihren religiösen Praktiken lediglich, weil die Schulordnung es ermöglicht, ihr Verhalten in Bezug auf die religiösen Praktiken streng zu regulieren, um die Laizität der Schule zu bewahren, die im Orientierungsgesetz Nr. 2003-17 vom 11. November 2003 über das nationale Bildungswesen in der Republik Benin vorgeschrieben ist. Allerdings beinhaltet die Schulordnung der technischen und beruflichen Sekundarschulen sowie der allgemeinbildenden Sekundarschulen (Schulordnung Nr. 156/ MESFTPRIJ /DC /SGM /IGPM /DEP /DET/ DESG / DAFOP /SA vom 31. März 2016) die folgenden Bestimmungen:

Artikel 8: Das Tragen von Hüten, Kopftüchern, Tragetüchern, Ornamenten und Phantasiegläsern ist innerhalb der Schule streng verboten.

Artikel 9: Extravagante Frisuren, Zöpfe und Make-up sind innerhalb der Schule streng verboten. Haare schneiden, sich kämmen, sich regelmäßig rasieren sind Teil der notwendigen Körpersauberkeit. Die Lernenden müssen daher nur natürliche oder einfach geflochtene Haare tragen.

Artikel 10: Körper- und Kleidungssauberkeit ist für alle Lernenden erforderlich.

Artikel 12: Die Lernenden sollen in der Klasse immer Schuhe tragen. Es ist verboten, barfuß innerhalb der Schule zu gehen.

Obwohl die Schulordnung in diesen Artikeln keine Religion nennt, kann man sehen, dass die verbotenen Kleider und Arten des Anziehens Unterscheidungszeichen von bestimmten Anhängern christlicher, muslimischer und animistischer Religionen sind. Der Sinn dieser Präskription ist es, eine Standardisierung der Schuluniform zu erreichen. Gleichzeitig versucht man dadurch zu vermeiden, dass die Lernenden ihre Religionszugehörigkeit zeigen, was sonst die Harmonie des Schullebens gefährden könnte. Offensichtlich will man die in Frankreich 
festgestellte Situation vermeiden, die A. Gagnieux im Jahr 2014 in seinem Artikel Les enjeux de l'interculturalité dans l'éducation wie folgt beschrieb:

\begin{abstract}
"Ainsi, l'école est devenue le lieu de conflits culturels et identitaires. Surtout dans les collèges et lycées des quartiers défavorisés. On relève des revendications d'élèves, souvent issus - mais pas toujours - de familles récemment immigrées qui méconnaissent la séparation fondamentale privélpublic instituée par le modèle français de lä̈cité. Il s'agit de la contestation des contenus d'enseignement (histoire-géo, SVT), voire des horaires des cours - des exigences alimentaires : hallal et casher... dont la riposte préconisée par le FN est le "repas laïque »-l'observance de certaines pratiques : ramadan, shabbat - le port d'insignes ou de vêtements religieux : kippa, foulard, croix - le refus de la mixité, ou des pressions à l'égard des filles, etc.,"
\end{abstract}

Der Kontext und die Schulrealitäten, die wir in der vorliegenden Arbeit untersuchen, unterscheiden sich von den oben beschriebenen französischen Realitäten. Die Lernenden bestreiten aus religiösen Gründen den Inhalt der Lehrpläne nicht. Ganz im Gegenteil: Obwohl $92 \%$ der Lernenden beispielweise Sex als heilig betrachten, finden viele Lernende (94\%, unterschiedener Religionen), dass Sexualerziehung gelehrt werden sollte. Die Mischung der Geschlechter ist auch kein zentrales Thema in beiden Zielschulen. Viele Lernende haben sich jedoch über Strafen für Taten beklagt, die in Übereinstimmung mit ihren religiösen Praktiken sind und in einem sehr geringen Verhältnis über Spitznamen, Spott und Diskriminierung in Bezug auf ihre Religionszugehörigkeit (siehe Ergebnisse der Umfrage im Anhang, S. xvii). Angesichts dieser Beschwerden ist es nachvollziehbar, dass die Lernenden ihre religiöse Identität deutlich zeigen wollen, aber sie sind mit den Anforderungen der Schulordnung konfrontiert, die unter anderem darauf abzielt, die Laizität der Schule in Benin beachten zu lassen. Ohne diese Regelung könnte das Bildungssystem religiösen Spaltungen und anderen Missständen ausgeliefert werden. Übrigens glaubt die Hälfte der befragten Lehrenden, dass die Lernenden diskriminierende Verhaltensweisen in Bezug auf ihren ethnischen und religiösen Hintergrund entwickeln.

Trotz präskriptiven Charakters der Schulordnung haben wir jedoch festgestellt, dass die Schulordnung in Bezug auf bestimmte kulturelle Situationen flexibel ist, um keine Kluft zwischen dem Schulleben, dem Familienleben oder dem sozialen Leben zu schaffen. Hier einige Beispiele: In einigen Ausnahmefällen dürfen die Lernenden-Adepten, abweichend von bestimmten Artikeln, religiöse Gegenstände tragen (siehe Artikel 97) und dürfen im Unterricht abwesend sein, um gelegentlich an religiösen Zeremonien teilzunehmen (siehe Artikel 64). Außerdem: Um die Integration mancher Adepten zu ermöglichen, ergreifen Anfang des Schuljahres die Schulbehörden bestimmte Maßnahmen. In CEG Tohouè sensibilisieren die Schulbehörden die Lernenden für ein würdevolles Verhalten gegenüber ihren Schulkameraden, die bestimmten Religionen angehören.

In diesem zweiten Teil der Präsentation der Ergebnisse können wir festhalten:

- Es besteht ein deutlicher Wunsch der Lernenden, ihre religiösen Identitäten zu zeigen.

- Die Artikel 8, 9, 10 und 12 der Schulordnung verbieten jegliches Zeigen der Religionszugehörigkeit. 
- Die Anwendung dieser Artikel trägt zum friedlichen Zusammenleben der verschiedenen Religionen in den Schulen bei.

- Die Schulordnung ist flexibel (siehe Artikel 64, 97) und erlaubt den Schulbehörden, Ausnahme- oder punktuelle Situationen zu lösen.

Aus dieser Analyse heraus, ergibt sich, dass ein gutes Management des Schullebens tatsächlich von der Berücksichtigung religiöser Faktoren abhängt.

\subsection{Berücksichtigung ethnolinguistischer Vielfalt als Kriterium eines guten Managements des Schullebens}

Wie bereits oben erwähnt, haben wir insgesamt 22 Ethnien unter den Lernenden und 13 unter den Lehrenden identifiziert, dazu 20 Muttersprachen unter den Lernenden und 17 unter den Lehrenden.

In CEG Tohouè werden folgende ethnische Gruppen unter den befragten Lehrenden verzeichnet worden: die Xwla, Aja, Gùn, Mina, Defi, Tori, Nago und unter den Lernenden die Xwla, Aja, Gùn, Mina, Defi, Nago, Yoruba, Sets, Maxi, Kotafon, Tofin. Wir haben festgestellt, dass die ethnischen Mehrheitsgruppen, nämlich die Defi, Gùn, Xwla (jeweils 6,7\%, 46,7\%, 20\% der Lehrenden und 26,7\%, 16,7\%, 10\% der Lernenden) und die ethnischen Minderheitsgruppen sowohl unter den Lehrenden als auch unter den Lernenden vertreten sind.

Die Muttersprachen der Lehrenden sind: Xwla, Gùn, Defi, Aja, Mina, Ife, Alada und die der Lernenden Xwla, Gùn, Defi, Maxi, Fon, Ewe, Yoruba, Nago und Təfin. Gùn wird sowohl von den Lehrenden (53,3\%) als auch von Lernenden (40\%) gesprochen.

Die bilaterale Repräsentativität der Ethnien und Sprachen ist einer der Indikatoren der kulturellen Vielfalt in einer Schule (vgl. Akkari, 2009: 88-89). Es kann ein erleichternder Faktor sein, um schwierige interkulturelle Situationen (Konflikte, Gruppen-Kohäsionsprobleme, Sensibilisierung, usw.) zu lösen. Der Grund dafür ist Folgendes: In einem interkulturellen Zwischenfall interpretieren die Konfliktparteien die Fakten zunächst in Bezug auf ihre ethnische und sprachliche Identität, bevor sie schließlich eine dezentrierende Haltung einnehmen. Übrigens haben wissenschaftliche Studien gezeigt, dass die gesprochene Sprache in den Augen der Individuen eines der bestimmenden Merkmale ihrer kulturellen Identität ist (vgl. Blanchet: 23).

Auch in CEG Banikanni sind folgende ethnische Gruppen unter den Lehrenden verzeichnet worden: die Fon, Baatonum, Nago, Boo, Waci, Waama und Idaaca und unter den Lernenden die Fon, Baatınum, Nago, Idaaca. Gùn, Yoruba, Maxi, Otamari, Dendi, Ifé, Nateni, Lokpa, xawsa, Saxwue und Pullo.

Die Muttersprachen, die bei den Lehrenden identifiziert wurden, sind: Maxi, Baatsnum, Dendi, Boo, Waci, Waama, Idaaca, Nago, Cabe und den Lernenden: Fon, Maxi, Baatonum, Dendi, Idaaca, Nago, Cabe, Gùn, Yoruba, Ditamari, Nateni, Lokpa, Pullo, Saxwue, Dyula und Yende.

In CEG Banikanni, die im Nordbenin liegt, ist eine Kuriosität bemerkbar: die Fon und Yoruba, ethnische Gruppen aus dem Südbenin, bilden die Mehrheit der befragten Lehrenden $(33,3 \%)$ und den Lernenden $(27,1 \%)$.

Diese starke Repräsentativität der südlichen ethnischen Gruppen in dieser Schule im Norden bestätigt, dass die beninische Schule zentralistisch gesprägt ist und dass die 
Einschreibung von Lernenden in den Schulen und die Einstellung von Lehrenden nicht nach ethnischen Kriterien erfolgt. Sie zeigt auch, dass eine interne Migration von der Bevölkerung besteht. Außerdem kann die beidseitige Repräsentativität ethnischer Gruppen unter den Lernenden und Lehrenden ein Vorteil für den Umgang mit interkulturellen Konfliktsituationen sein. Diese Erkenntnis soll nicht heißen, dass wir hier das Management des Schullebens auf die Ethnisierung stützen, ein Begriff, den F. Lorcerie (2003: 24) wie folgt definiert:

\begin{abstract}
"[...] le processus par lequel l'imputation ou la revendication d'appartenance ethnique devient un référent déterminant (fondamental, englobant, dominant, voire exclusif) de l'action et dans l'interaction. Les acteurs sociaux ethnicisent une relation, une situation, un cadre, lorsqu'ils lui appliquent une grille de lecture en termes d'appartenance ethnique, c'est-à-dire dès lors qu'ils pensent et interagissent comme si les conditions sociales étaient déterminées, significativement ou totalement, par l'appartenance ethnique.,,
\end{abstract}

Vielmehr soll hier lediglich betont werden, dass Ethnisierung ein latentes Phänomen in den beninischen Schulen ist. Die Felduntersuchungen bestätigen dies: 63,3\% der befragten Lehrkräfte glauben, dass historischer Streit zwischen ethnischen Gruppen sich auf den Zusammenhalt der Klassengruppen auswirken und 13,3\% sagen, dass sie wegen dieses historischen Streits gegen manche Lernenden bestimmter ethnischen Herkunft Groll hegen. Außerdem behauptet die Hälfte der befragten Lehrkräfte - wie bereits angesprochen -, dass die Lernenden diskriminierendes Verhalten im Zusammenhang mit ihrer ethnischen Herkunft und ihren religiösen Vorstellungen haben.

Die Lernenden ihrerseits erwähnten einige Akte ethnischer Diskriminierung und frustrierende Praktiken:

- Die Xwla unterscheiden sich von den Defi,

- die Etikettierung bestimmter ethnischer Gruppen als Hunde-, Garifresser oder Kuhmilchtrinker,

- Spitznamen im Zusammenhang mit religiösen und ethnischen Zugehörigkeiten,

- Verbot, die Muttersprache zu sprechen,

- Tabubrüche (Ein Aufseher hätte ein Mädchen geohrfeigt, was nach der Sitten seiner Familie verboten ist).

Darüber hinaus fühlt sich ein kleiner Teil der Lernenden (3\% der Lernenden bestätigen es kategorisch; $15 \%$ weniger kategorisch) von ihren Klassenkameraden und Lehrenden wegen ihres religiösen, ethnischen und kulturellen Hintergrunds verworfen oder abgelehnt. Ebenso gibt ein geringer Anteil der Lernenden (kategorisch: 7\%, weniger kategorisch: 17\%) an, dass die Lehrenden im Unterricht schlecht über ihre ethnische Zugehörigkeit, Sprache und Eltern sprechen. 


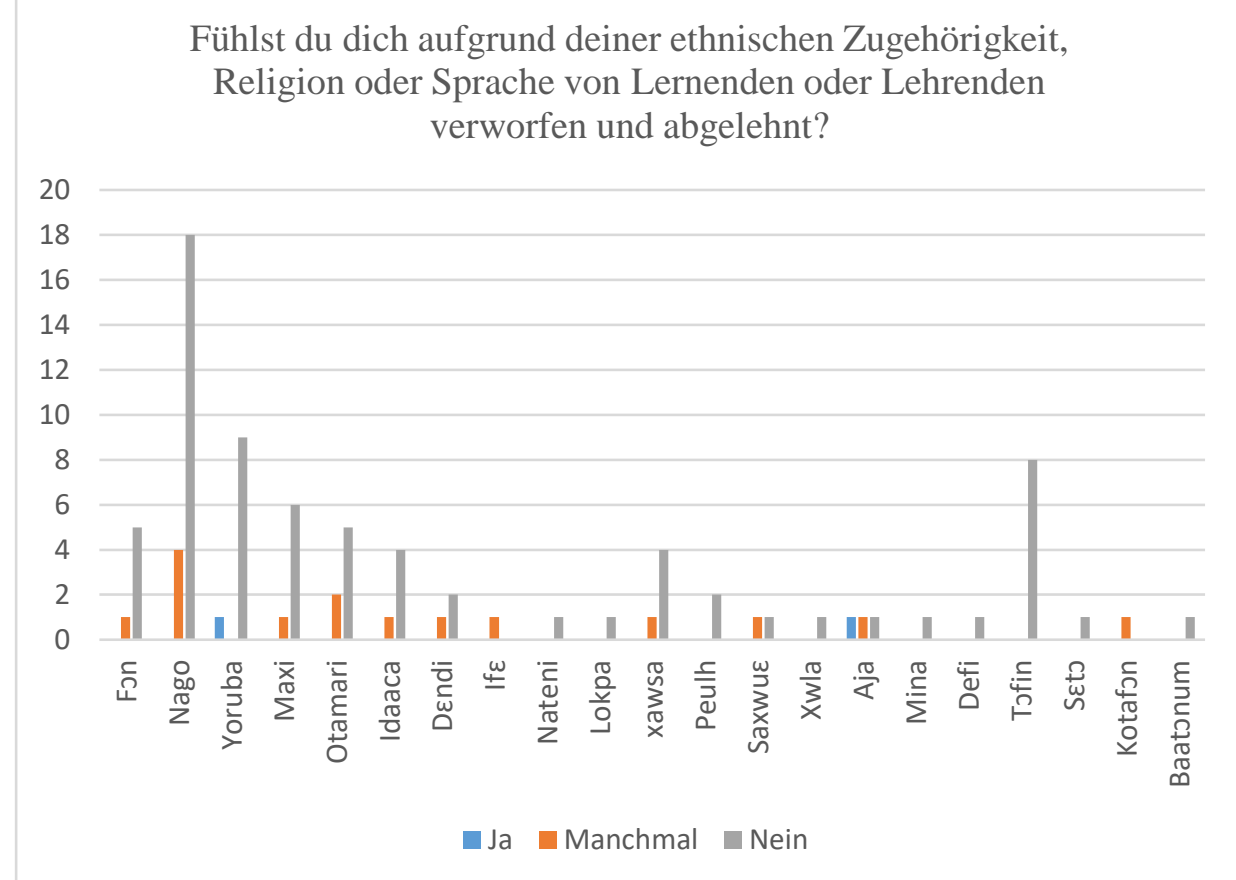

Abbildung 2: Ergebnis der Frage 16 an die Lernenden

Wenn die Beziehungen zwischen den Lernenden und Lehrenden sich ethnisieren, erfahren meistens die ethnischen Minderheiten ethnische Diskriminierung. Die Opfer bevorzugen in diesem Fall, angesichts des Machtverhältnisses zu schweigen und erleben diese Diskriminierung als Frustration.

Aber auch ernährt sich ethnische Diskriminierung in den Schulen meistens von historischen Streiten, zum Beispiel von denen zwischen den Tori und den Fon von Abomey, den Fon und den Idaaca, den Baatsnum und den Somba etc.

Auf der anderen Seite können sich die Beziehungen zwischen Lernenden, Lehrenden und sogar zwischen Lehrenden und Lernenden aufgrund freundschaftlicher Bande ethnisieren. Zum Beispiel haben sich die Batonuum und den Nago aus der Gegend von Tchaourou seit geraumer Zeit angefreundet. Diese freundschaftliche Bande zwischen den beiden ethnischen Gruppen konkretisiert sich durch das Praktizieren von der scherzhaften Verwandtschaft (der joking relationship). Die scherzhafte Verwandtschaft ist nach Wikipedia eine typische westafrikanische soziale Praktik, die berechtigt, bestimmte Kategorien von Familiennamen und manchmal sogar Mitglieder der gleichen Familie (wie entfernte Cousins) oder die Mitglieder bestimmter ethnischer Gruppen unter ihnen zu verspotten oder zu beleidigen, und dies ohne Folgen. Diese verbalen Zusammenstöße sind in der Tat Mittel der sozialen Entspannung. Immer nach Wikipedia müssen die Parteien, die sich an dieser sozialen Praktik beteiligen, Beziehungen der Gastfreundschaft, der gegenseitigen Hilfe, der Loyalität, gegenseitige Anerkennung beachten und vermeiden, sich selbst zu schaden. Die scherzhafte Verwandtschaft gilt für fast alle Gesellschaftsstrukturen: Familie, Altersgruppe, Heiratsverbündete, benachbarte Dörfer, benachbarte Gebiete und benachbarte ethnische Gruppen.

Wir halten am Ende dieses Abschnitts fest, dass die beiden im Mittelpunkt der vorliegenden Studie stehenden Schulen aus einer Vielfalt von ethnischen Gruppen bestehen. 
Viele Lehrenden und Lernenden stammen aus denselben ethnischen Gruppen. Dies kann ein kompromissfördernder Faktor für interkulturelle Verhandlungen sein, falls es in den Schulen zu Konflikten kommt. Außerdem zeigen die Angaben der Befragten, dass ethnische Diskriminierung in beiden Schulen besteht.

Seit der Kolonialzeit wurden die Muttersprachen vom Unterricht zugunsten der französischen Sprache ausgeschlossen. Bei der Diagnose der Probleme, die die beninische Schule untergraben, erklärte H. B. C. Capo: "L'école au Bénin a toujours exclu les langues nationales. A l'origine de l'école béninoise, était l'école coloniale qui interdisait, pour des raisons idéologiques évidentes, l'usage des "langues autochtones", même à des fins d'illustration ». (La Nation vom 4. 3. 2014).

In der Tat, war die Unterrichtssprache Französisch, weil es eines der Ziele von französischen Missionaren in Afrika war. T. M. C. Gbetie (2015:88) stellt dies klar unter Berufung auf G. Chatelain, der sagt: " Des missionnaires, déjà pendant la traite des Noirs se sont installés un peu partout en Afrique en vue d'une part, d'enseigner le français qui serait la base de tout enseignement mais aussi et surtout parce qu'il est un meilleur instrument de civilisation ». Diese Situation dauerte lange in Benin, bis befugte Stimmen für einen Paradigmenwechsel plädierten. Der ehemalige Präsident Mathieu Kerekou hat im Rahmen des Rede-Programms vom 30. November 1972 einen Appell zur Aufwertung der Nationalsprachen und zur Rehabilitierung der beninischen Kultur ausgerufen (vgl. Gbetie, 2015:95). Ein umfangreiches Alphabetisierungsprogramm wurde dann gestartet. Dieses Programm wurde leider in den 1990er Jahren mit der Nationalkonferenz der lebendigen Kräfte unterbrochen, die eine neue Lehrplanorientierung verlangten. Aber in letzter Zeit ist der Wille des Staates entstanden, das Lehren der Nationalsprachen in den Schulen wieder einzuführen, und zwar gemäß dem Artikel 8 des Orientierungsgesetzes (la loi d'orientation), der verfügt:

„L'enseignement est dispensé principalement en français, en anglais et en langues nationales. Les langues nationales sont utilisées d'abord comme matière et ensuite comme véhicule d'enseignement dans le système éducatif. En conséquence, l'Etat doit promouvoir les recherches en vue de l'élaboration des instruments pédagogiques pour l'enseignement des langues nationales aux niveaux maternel, primaire, secondaire et supérieur."

Strukturell gesehen gibt es konkrete Maßnahmen in dieser Richtung. Zum Beispiel wurden Alphabetisierungsdienste bei der DIPIQ und der DDESTFP (vgl. Artikel 13 und 14 des Ministerialerlasses Nr. 108 / MESTFP / DC / SGM / DIPIQ / SA / 059SGG17 vom 27. Oktober 2017 über die Zuständigkeiten, Organisation und Funktionsweise der Direktion für die pädagogischen Inspektion, Innovation und Qualität und Artikel 9 und 10 der Ministerialerlasses Nr. 145 / MESTFP / DC / SGM / DDESTFP / DIPIQ / SA / 103SGG17 vom 29. Dezember 2017 über die Zuständigkeiten, Organisation und Funktionsweise der Directions Départementales des Enseignements Secondaire, Technique et de la Formation Professionnelle) gegründet. Aber in den Schulen wird das Lehren der Nationalsprachen trotz des ELAN-Projekts nur langsam Wirklichkeit. Schlimmer noch, das Verbot der Verwendung von Muttersprachen durch Lernende in Klassenräumen scheint noch aktuell zu sein. Die befragten Lernenden, indem sie Beispiele für diskriminierende Handlungen in Bezug auf ethnische Zugehörigkeit und 
Religionszugehörigkeit in ihren Schulen geben, haben auch das Verbot der Verwendung von Muttersprachen genannt. Es wird aber wissenschaftlich bewiesen, dass beim Lernen im Allgemeinen und im Besonderen beim Erlernen von Fremdsprachen der Lernende von seinen Vorkenntnissen ausgeht, um neues Wissen bzw. neue Kompetenzen zu entwickeln. M-P. Perdereau-Bilski (2001:143) drückt dieses pädagogische Prinzip folgendermaßen aus:

"Il est commun de remarquer que l'enfant, même en langues, n'arrive pas vierge de toutes compétences et savoirs sur les aspects de ces langues. [...] On ne peut ignorer ce processus sous peine de susciter un certain déséquilibre affectif, cognitif et surtout culturel. La culture enfantine est une composante essentielle dans la conduite d'un tel apprentissage social, culturel et langagier."

Für den Fremdsprachenunterricht ist es daher notwendig, dass die Curricula erneut entwickelt werden (56\% der befragten Lernenden und 73,3\% der befragten Lehrkräfte geben an, dass sie ihre Werte und kulturellen Praktiken nie im Lehrplan finden). Gleichzeitig sollen die Lehrenden die soziokulturellen Realitäten und die Muttersprachen der Lernenden berücksichtigen, ohne die das Lernen nicht möglich ist. Perdereau-Bilski (2001:147) behauptet in diesem Zusammenhang : "Les stratégies interlinguales ont mis en évidence que la langue maternelle agit comme une bouée par laquelle passe toute la compréhension et l'expression visées ».

Er erklärt, dass die Verwendung der Muttersprache dazu dient, Interesse, Kontakt und Kommunikation gleichzeitig aufrechtzuerhalten (vgl. M-P. Perdereau-Bilski 2001:148). Ihm zufolge soll der Lehrende in der Lage sein, die Verwendung der Muttersprache durch die Lernenden in ihren mündlichen und schriftlichen Produktionen (in Fremdsprachen) zu interpretieren. M-P. Perdereau-Bilski (2001:148) zitiert in diesem Zusammenhang Daniel Moore wie folgt:

Contrairement aux idées reçues, le recours à la langue maternelle dans la production d'énoncés en langue autre, n'est pas simple indicateur d'incompétence ou de confusion de langues mais marque la mise en œuvre de stratégies intéressantes, notamment pour appeler l'attention de l'enseignant et l'amener à répondre à la sollicitation. L'alternance peut fonctionner comme un savoir sollicité qui est un processus dynamique d'acquisitions de nouvelles formes, de nouveaux savoirs en langue maternelle et entre dans un schéma interactionnel facilitateur.

Die Ergebnisse der Studie von F. Wantz-Bauer (2001:44) zeigen auch, wie wichtig die Verwendung der Muttersprache beim Fremdsprachenlernen ist. Er führte Interviews mit Lernenden hinsichtlich ihrer Erwartungen an die Kompetenz des Lehrenden in ihrer Muttersprache und hinsichtlich der Verwendung der Muttersprache im Klassenzimmer, einerseits durch den Lehrenden, andererseits durch die Lernenden selbst. Die Lernenden haben deutlich erklärt, dass sie es bevorzugen, dass der Lehrende ihre Muttersprache kennt und sie angemessen im Unterricht verwendet. Die im Rahmen der vorliegenden Studie befragten Lernenden (89\%) geben an, dass die Verwendung der Muttersprache manchmal es einfacher macht, Fremdsprachen zu lernen. Die meisten befragten Lernenden (79\%) erwähnten jedoch nicht die pädagogischen Aktivitäten, bei denen sie auf Schwierigkeiten stoßen. Die wenigen genannten Schwierigkeiten sind: Verständnisschwierigkeiten (8\%), Ausspracheschwierigkeiten (6\%), Schwierigkeiten beim Schreiben (4\%), Schwierigkeiten beim Sprechen (3\%). Diese 
Schwierigkeiten zeigen, warum sich die Mehrheit der Lernenden für Grammatik mehr interessieren (Nur 1\% der befragten Lernenden geben an, dass sie grammatikalische Schwierigkeiten haben). Angesichts dieser Fakten können wir bestätigen, dass das gute Management des Schullebens von der Berücksichtigung ethnolinguistischer Faktoren abhängt. Die Schulordnung erwähnt deutlich keine Maßnahmen im Falle eines ethnischen Konflikts, aber sie überlässt in ihrem Artikel 139 den Schulbehörden, jegliche andere Situationen einzuschätzen.

\subsection{Berücksichtigung der Genderfrage als Kriterium eines guten Managements des Schullebens}

Die Genderfrage, Gender im Sinne von sozialem Geschlecht, das von gesellschaftlichen Rollenvorstellungen des Männlichen und des Weiblichen geprägt ist, wird in der vorliegenden Studie aus der Sicht der Geschlechtsmischung bzw. der Koedukation betrachtet. Letztere wird als die gemeinsame Unterweisung von Mädchen und Jungen in derselben Klasse begriffen.

Die beninische Schule ist wie in vielen Ländern von der Koedukation geprägt. Die Statistiken zur Anzahl der befragten Lernenden verdeutlichen dies recht gut: Es gibt 64 Jungen und 36 Mädchen in den untersuchten Abschlussklassen. Die geringe Anzahl von Mädchen erklärt sich durch die niedrige Einschulungsquote der Mädchen auf nationaler Ebene. Um dieses Problem der geringen Einschulungsquote von Mädchen im Bildungssystem zu beheben, hat der beninische Staat die Befreiung von Schulgebühren für alle Mädchen in der Primarschule und in der Sekundarstufe 1 eingeführt. Eine weitere Maßnahme war die Schaffung von Mädchengymnasien und somit die Schaffung von monoedukativen Schulen. Es gibt sieben staatliche Mädchengymnasien in Benin: das Mädchengymnasium Toffa $1^{\mathrm{er}}$ in Porto-Novo, das Mädchengymnasium in Ouidah, das Mädchengymnasium in Lokossa, das Mädchengymnasium Houfon in Abomey, das Mädchengymnasium in Parakou, das Mädchengymnasium und das Militärmädchengymnasium in Natitingou.

Einige Länder auf der Welt haben eine lange Tradition mit der monoedukativen Schule. In der Zusammenfassung seines Artikels, aus dem Englischen ins Französische von Marcel Rieu übersetzt, nennt Emer Smyth (2010) die Länder, in denen es nur monoedukative Schulen gibt. Es sind Australien, Neuseeland und Irland. Das Vereinigte Königreich und die Vereinigten Staaten entscheiden sich zunehmend für monoedukative Klassen, hauptsächlich um die Bildungsergebnisse zu verbessern. Laut Smyth (2010:57-58) sind die Gründe für die Schaffung von monoedukativen Klassen die folgenden: das Verhalten von Jungen, die im Unterricht die Aufmerksamkeit auf sich richten lassen, das Konzentrationsdefizit von Teenagern, die mit Lernenden des anderen Geschlechts in derselben Klasse sitzen und die Tatsache, dass Schulen als Ort der Konstruktion von Männlichkeit und Weiblichkeit dienen. Bei der Analyse von den Schlussfolgerungen der unterschiedlichen Studien gelangt Smyth (2010: 57) zum folgenden Standpunkt: "Il semble qu'il y ait peu de consensus sur le fait de savoir si une éducation non mixte est plus avantageuse pour les filles ou pour les garçons en termes de résultats scolaires ». Im beninischen Kontext haben wir einige Erfolgsquoten bei den staatlich organisierten Abschlussprüfungen des Schuljahres 2017-2018 in einigen geschlechthomogenen Gymnasien sammeln können:

- $\quad$ Lycée Toffa $1^{\mathrm{er}}$ : BEPC (33,51\% ), BAC ( $\left.42,40 \%\right)$ 
- Lycée des Jeunes Filles de Ouidah : BEPC (57,97\%), BAC (50,98\%)

- Lycée des Jeunes Filles de Lokossa : BEPC (65\%), BAC (62,50\%)

- Lycée Houfon des Jeunes Filles d'Abomey : BEPC (33,10\%)

- Lycée des Jeunes Filles de Parakou : BEPC (31, 25\%), BAC (26, 92\%)

- Lycée des Jeunes Filles de Natitingou : BEPC (36,17\%), BAC (24,14\%)

- Lycée Militaire des Jeunes Filles de Natitingou : BEPC (100\%), BAC (100\%)

Beim Vergleich der obenstehenden Erfolgsquoten mit denen von CEG Banikanni (BEPC 17,17\%; BAC 32,68\%) und CEG Tohouè (BEPC: 17,43\%; BAC : 27,27\%) stellen wir fest, dass die geschlechthomogenen Gymnasien in dem Schuljahr 2017-2018 bessere Schulergebnisse erzielt haben. Es ist aber schwer, daraus zu schlussfolgern, dass in Benin die geschlechtshomogenen Schulen leistungsfähiger sind, weil es andere Faktoren gibt, die diese besseren Ergebnisse erklären könnten, zum Beispiel die geringe Anzahl von Lernenden in den Abschlussklassen und somit die effiziente Betreuung, von denen diese Lernenden profitieren.

Darüber hinaus haben wir auf der Disziplinarebene einige Gründe für die Bestrafung der Lernenden an dem Mädchengymnasium Toffa $1^{\text {er }}$ gesammelt, um einen Vergleich zu machen. Die wiederkehrenden Gründe sind:

- Sich verspäten,

- Abwesenheit an den Kursen,

- Ungemachte Übungen,

- In der Klasse Lärm machen oder schwatzen,

- Schlendern,

- Versäumen, die Klasse aufzuräumen,

- In der Klasse essen,

- Kopftuch tragen,

- Handy bei sich haben oder Benutzung des Handys.

Insgesamt sind die oben erwähnten Bestrafungen die gleichen wie in den anderen koedukativen Schulen in Benin. Eigentlich regelt die gleiche Schulordnung ( $\left(\mathrm{N}^{\circ} 156 /\right.$ MESFTPRIJ /DC /SGM /IGPM /DEP /DET/ DESG / DAFOP /SA vom 31. März 2016) das Schulleben sowohl in Mädchengymnasien als auch in den koedukativen Sekundarschulen. Im Grunde genommen gibt es keinen grundlegenden Unterschied auf der Leistungs- und Disziplinebene zwischen den monoedukativen und koedukativen Sekundarschulen in Benin. Übrigens: Abgesehen von den sieben oben genannten geschlechtshomogenen Gymnasien werden alle öffentlichen Sekundarschulen nach dem Prinzip der Koedukation geschaffen.

Aber wie wirkt sich die Koedukation auf das Schulleben aus, welche das Prinzip der Geschlechtergleichheit in den Schulen respektieren möchte? Um es herauszufinden wurden die Lernenden gefragt, ob ihre Altersgenossen sie in Spiel- oder Lerngruppen leicht akzeptieren. 32 von 36 Mädchen antworteten mit Ja. Die vier restlichen Mädchen erwähnen nicht die Gründe, warum sie diskriminiert werden. 30 Mädchen bestätigen die Wichtigkeit der Schule und 33 (auch 46 Jungen von 64) geben an, dass Mädchen zur Schule gehen müssen. Es kann infolgedessen behauptet werden, dass die Mädchen in CEG Banikanni und CEG Tohouè gut im Schulleben integriert sind. Dies bestätigt den Befund von E. Smyth (2010: 48) 
«Tout en s'intéressant aux processus éducatifs que cela implique, un certain nombre d'études ont évalué l'importance de l'influence d'une éducation non mixte sur le plan du développement personnel et social chez les jeunes. Il semble y avoir un consensus général sur le fait que les élèves des écoles mixtes, filles et garçons confondus, voient leur école de façon plus positive et sont plus positifs aussi sur les différents aspects de leur enseignement. »

Es gibt jedoch einige negative Auswirkungen, die die Geschlechtsheterogeneität auf das Schulleben haben könnte, wie M. Duru-Bellat (2010:198) in ihrem Artikel Ce que la mixité fait aux élèves hervorhebt: «[...] le quotidien des classes est le théâtre d'interactions entre enseignants et élèves, et entre élèves profondément marquées par les représentations sociales du masculin et du féminin».

In der Tat beobachten wir am häufigsten in der Sekundarstufe 2, dass die Jungen zurückhaltend sind, Sauberkeitsdienste zu tun, vor allem das Klassenaufräumen. Sie behaupten oft, dass diese Aufgabe traditionell Mädchen vorbehalten ist, deshalb weigern sie sich an dem Aufräumen teilzunehmen. In diesem Fall wird die gesamte Gruppe, Mädchen wie Jungen, die das Klassenzimmer aufräumen soll, bestraft. Auch wird während der Schulfeiern das Kochen oft den Mädchen anvertraut. Es gibt andere Formen der Rollenvorstellung des Männlichen und Weiblichen in der Schule: Die meisten Mädchen bevorzugen es, ausschließlich miteinander zu spielen, die Jungen auch so. Außerdem weigern sich manche Jungen aus religiösen Gründen neben Mädchen zu sitzen.

Andere Probleme können hier erwähnt werden, die durch die Koedukation verschärft werden, wie zum Beispiel das Unbehagen, das die Mädchen erfahren, um ihre Privatsphäre zu bewahren (Mangel an Toiletten, Latrinen, Umkleideräumen, usw.), die sexuelle Belästigung, das Anschauen von Pornographie-Filmen. Darüber hinaus machen die meisten Lernenden in den Abschlussklassen die Adoleszenz durch, wie die Altersgruppen der befragten Lernenden es zeigen:

Tabelle 1: Altersgruppen der Lernenden

\begin{tabular}{|l|c|c|}
\hline Alter der befragten Lernenden & Anzahl & Prozentsatz \\
\hline 16 & 3 & $3 \%$ \\
\hline 17 & 4 & $4 \%$ \\
\hline 18 & 23 & $23 \%$ \\
\hline 19 & 15 & $15 \%$ \\
\hline 20 & 20 & $20 \%$ \\
\hline 21 & 11 & $11 \%$ \\
\hline 22 & 10 & $10 \%$ \\
\hline 23 & 5 & $5 \%$ \\
\hline 24 & 5 & $24 \%$ \\
\hline 25 & 2 & $25 \%$ \\
\hline 26 & 1 & $26 \%$ \\
\hline Fehlende Antworten & 1 & $1 \%$ \\
\hline Gesamt & 100 & $100 \%$ \\
\hline
\end{tabular}

Die Adoleszenz, eine Zeit des psychologischen Umbruchs, ist durch spezifische Verhaltensweisen gekennzeichnet, die manchmal zu Disziplinproblemen führen. In dieser Zeit manifestieren junge Menschen ihre Männlichkeit bzw. ihre Weiblichkeit. Die Jungen versuchen, 
ihre Männlichkeit und Kräftigkeit zu demonstrieren. Die Mädchen ihrerseits versuchen, die Jungen zu verführen, indem sie sich um ihre körperliche Erscheinung kümmern und der Mode anpassen. Diese verschiedenen juvenilen Manifestationen führen manchmal zu abweichendem Verhalten: sprachlicher Entgleisung, ungewollten Schwangerschaften, Suche nach leichten Noten, Disziplinlosigkeit und Aggressivität. Mit K. Flaake (2006:39) stellen wir fest: "In gemischtgeschlechtlichen Kontexten kommen Männlichkeits- und Weiblichkeitsinszenierungen mit besonderer Vehemenz zum Tragen - sind sie doch immer auch auf das jeweilige andere Geschlecht bezogen."

Am Ende der Diskussion in diesem Abschnitt halten wir fest, dass die beninische Schule geschlechtsgemischt ist. Die Koedukation führt dazu, dass das Schulleben stark durch soziale Repräsentationen des Männlichen und des Weiblichen geprägt ist. Sie verursacht einige Beziehungsprobleme zwischen den zwei Geschlechtern, die Aufsichtsbehörden zu lösen versuchen. Aus diesen Erwägungen können wir die Hypothese bestätigen, dass das gute Management des Schullebens von der Berücksichtigung der Genderfrage abhängt.

\section{Schlussfolgerung}

Ziel der Arbeit war es, die Relevanz eines guten Managements der kulturellen Vielfalt für die Verbesserung der pädagogischen Handlung und des Schullebens in zwei allgemeinbildenden Sekundarschulen zu untersuchen. Auf der einen Seite konnte gezeigt werden, dass CEG Banikanni und Tohouè ethnisch, religiös, sprachlich heterogen und geschlechtsgemischt sind. Die Studie hat auch gezeigt, dass das richtige Management der kulturellen Vielfalt von der Berücksichtigung religiöser, ethnischer, sprachlicher und geschlechtsspezifischer Faktoren abhängt. Diese Ergebnisse bestätigen, dass die kulturelle Vielfalt a priori ein Vorteil für die pädagogische Handlung und für das Schulleben ist. Aber sie kann ein Engpass sein, wenn sie nicht gut gemanagt wird. Übrigens verlassen sich Verwaltungsbehörden auf die Schulordnung, um sie managen zu können. In Anbetracht dieser Feststellungen kann nicht behauptet werden, dass die kulturelle Vielfalt in beninischen allgemeinbildenden Sekundarschulen ein Hindernis für eine gute Klassenführung ist.

\section{Conflict of Interest Statement}

The authors declare no conflicts of interests.

\section{About the Authors}

Dr. Vincent Atabavikpo, actuel Doyen de la Faculté, des Lettres, Langues, Arts et Communication (FLLAC), est Enseignant-chercheur, Maître de Conférences en Germanistique à l'Université d'Abomey-Calavi en République du Bénin. Il est écrivain d'expression allemande et auteur de plusieurs ouvrages et articles scientifiques.

L'Inspecteur de l'Enseignement Secondaire, Sèlognon Nonvide, est diplômé de l'Ecole de Formation des Personnels d'Encadrement de l'Education Nationale (EFPEEN) de Porto-Novo. Professeur certifié de l'ENS de Porto-Novo et titulaire d'une Maîtrise d'Allemand à l'UAC, il enseigna l'allemand au cours secondaire et a été parallèlement chargé de cours au Département 
des Etudes Germaniques à l’Université Nationale de Calavi. A l'Université de Bayreuth (19961997), l'ancien boursier du DAAD fit des recherches en littérature allemande qui aboutissent à la rédaction de son mémoire de Maîtrise «Funktion des Geldes in Dürrenmatts Der Besuch der alten Dame ». Il fit ses études primaires (1975-1981) et secondaires (1981-1990) au Togo.

\section{Literatur}

Ahlring Ingrid 2002: "Vielfalt als Chance», in Ingrid AHLRING: Differenzieren und Individualisieren, Braunschweig: Westermann, S. 8-12.

Akkari Abdeljalil (2009) : Introduction aux approches interculturelles en éducation. Genève : Université de Genève, Faculté de psychologie et des sciences de l'éducation, $<$ http://archive-ouverte.unige.ch/unige:21763 -Zugriff am 4. 6. 2018>

Blanchet Philippe: L'approche interculturelle comme principe didactique et pédagogique structurant dans l'enseignement/apprentissage de la pluralité linguistique. $<$ https://fr.scribd.com/document/259175524/blanchet-pdf -Zugriff am 12. 3. 2017>

Duru-Bellat Marie (2010) : Ce que la mixité fait aux élèves. In : Revue de l'OFCE, $\mathrm{N}^{\circ} 114$ - juillet

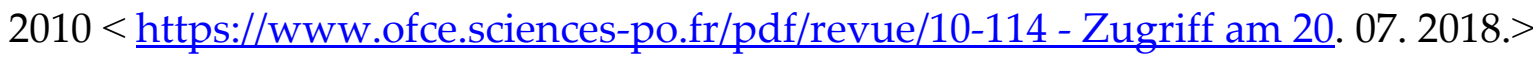

Flaake Karin (2006): „Geschlechterverhältnisse - Adoleszenz - Schule. Männlichkeits- und Weiblichkeitsinszenierungen als Rahmenbedingungen für pädagogische Praxis. Benachteiligte Jungen und privilegierte Mädchen? Tendenzen aktueller Debatten“. In Jösting, Sabine \& Seemann Malwine (Hrsg.): Gender und Schule. Geschlechterverhältnisse in Theorie und schulischer Praxis. O. O u. V. S. 30-45.

Gagnieux Alain (2014): Les enjeux de l'interculturalité dans l'éducation. $<$ http://www.alaingagnieux.fr/les-enjeux-de-linterculturalite-dans-leducation-2/ - Zugriff am 25. 7. 2017>

GBETIE Théodore Marc Comlan (2015): Législation et administration scolaires en République du Bénin. Porto-Novo: EPA/CERADE.

Hounkpati B. C. Capo La Nation du 4 mars 2014. <http://news.acotonou.com/h/19620.html Zugriff am 15. 3. 2018>

Lorcerie Françoise: L'école et le défi ethnique. ESF Editeur; INRP, 2003, Actions sociales/Confrontations, Eric Debarbieux, 2-7101-1592.1. <https://hal.archivesouvertes.fr/hal-00819790/document.pdf - Zugriff am 8. 7. 2018>

Maalouf Amine (1998) : Les Identités meurtrières. Paris : Grasset.

Perdereau-Bilski, Marie-Patricia (2001) : «Des langues-cultures autres à la culture de sa langue maternelle: vers un plurilinguisme culturel dès la petite enfance ». In CASTELLOTI, Véronique: D'une langue à d'autres: Pratiques et représentations. Rouen : Publications de l’Université de Rouen, p.129-150.

Smyth Emer (2010) : «Non-mixité à l'école : que nous dit la recherche actuelle »? In : Revue française de pédagogie, $N^{\circ}$ 171, avril-mai-juin $2010<$ http://ife.enslyon.fr/publications/edition-electronique/revue-francaise-de-pedagogie/RF171-6.pdf Zugriff am 20. 07. 2018> 
Wantz-Bauer Frédérique (2001): «Le statut de la langue maternelle en cours de langue étrangère : Points de vue des apprenants ». In CASTELLOTI, Véronique: $D^{\prime}$ une langue à d'autres: Pratiques et représentations. Rouen: Publications de l'Université de Rouen, p.3957. 
Sèlognon Nonvide, Vincent Atabavikpo

KULTURELLE HETEROGENITÄT ALS HERAUSFORDERUNG

IN BENINISCHEN ALLGEMEINBILDENDEN SEKUNDARSCHULEN

Creative Commons licensing terms

Author(s) will retain the copyright of their published articles agreeing that a Creative Commons Attribution 4.0 International License (CC BY 4.0) terms will be applied to their work. Under the terms of this license, no permission is required from the author(s) or publisher for members of the community to copy, distribute, transmit or adapt the article content, providing a proper, prominent and unambiguous attribution to the authors in a manner that makes clear that the materials are being reused under permission of a Creative Commons License. Views, opinions and conclusions expressed in this research article are views, opinions and conclusions of the author(s). and European Journal of Literature, Language and Linguistics Studies shall not be responsible or answerable for any loss, damage or liability caused in relation to/arising out of conflicts of interest, copyright violations and inappropriate or inaccurate use of any kind content related or integrated into the research work. All the published works are meeting the Open Access Publishing requirements and can be freely accessed, shared, modified, distributed and used in educational, commercial and non-commercial purposes under a Creative Commons Attribution 4.0 International License (CC BY 4.0). 